\title{
Simultaneous Determination of Luteoloside, Apigetrin, And Hesperidin In Rat Plasma By UHPLC-MS/MS: Application to A Comparative Pharmacokinetic Investigation After Oral Administration Of Schizonepeta Tenuifolia Aqueous Extract With And Without Its Volatile Oil
}

Siting Liu

Nanjing Children's Hospital: Nanjing Medical University Second Affiliated Hospital

Yulan Jiang

Nanjing University of Chinese Medicine

Mingqiu Shan ( $\nabla$ shanmingqiu@163.com )

Nanjing University of Chinese Medicine https://orcid.org/0000-0002-3029-7074

Sheng Yu

Nanjing University of Chinese Medicine

Fangfang Cheng

Nanjing University of Chinese Medicine

Peidong Chen

Nanjing University of Chinese Medicine

Beihua Bao

Nanjing University of Chinese Medicine

Yudan Cao

Nanjing University of Chinese Medicine

Li Zhang

Nanjing University of Chinese Medicine

Qinan Wu

Nanjing University of Chinese Medicine

\section{Research}

Keywords: UHPLC-MS/MS, Schizonepeta tenuifolia, Pharmacokinetics, Rat plasma

Posted Date: October 16th, 2020

DOI: https://doi.org/10.21203/rs.3.rs-90750/v1

License: @ (i) This work is licensed under a Creative Commons Attribution 4.0 International License. Read Full License 


\section{Abstract}

Background: Schizonepeta tenuifolia Briq. (ST) has been used as an aromatic exterior-releasing medicine in clinical practice for thousands of years in China. Previous researches have revealed both volatile oil (STVO) and aqueous extract (STAE) from ST showed significant pharmacological activities. However, the influence between each other was still unknown.

Methods: This study was designed to compare the pharmacokinetic profiles of three main flavonoids (luteoloside, apigetrin, and hesperidin) in STAE to illustrate the influence of STVO. So, an ultra-flow liquid chromatography-tandem mass spectrometry (UHPLC-MS) method was established to quantitatively analyze the three absorbed ingredients in the plasma of healthy rats. Biological samples were analyzed on an Agilent Eclipse Plus $\mathrm{C}_{18}$ column $(3.0 \mathrm{~mm} \times 150 \mathrm{~mm}, 3.5 \mu \mathrm{m})$ with gradient mobile phase (containing $0.2 \%$ formic acid and acetonitrile) at a flow rate of $0.8 \mathrm{~mL} / \mathrm{min}$. All the analytes and quercitrin (IS) were investigated with an electrospray ionization source (ESI) using multiple-reaction monitoring (MRM) in negative ionization mode.

Results: This quantitative method showed good linearities $(r \geq 0.9995)$ and the lower limits of quantification were $0.590 \sim 1.19 \mathrm{ng} / \mathrm{ml}$. The intra- and inter-day precisions ranged 3.47 10.45\% and $4.29 \sim 11.28 \%$ for the three analytes. The mean extraction recoveries were in the range of $77.41 \sim 109.79 \%$ and the average matrix effects were within $83.41 \sim 112.67 \%$. The validated method has been fully applied to compare the pharmacokinetic parameters of the three flavonoid glycosides in rat plasma after oral administration of STAE and STAE+STVO. In comparison of luteoloside, apigetrin, and hesperidin in STAE group, it was found that different degree of increasing existed for the time to reach the maximum concentration $\left(T_{\text {max }}\right)$, elimination half-life time $\left(T_{1 / 2}\right)$, the area under the concentration curves $\left(A_{U} C_{0 \rightarrow t}\right.$ and $\left.\mathrm{AUC}_{0 \rightarrow \infty}\right)$ and the maximum concentrations $\left(\mathrm{C}_{\max }\right)$ in STAE+STVO group.

Conclusions: As can be seen from above, STVO could improve the absorption and bioavailability of the three analytes. These findings would provide some active and strong basis of safe clinical application for ST and further exploitation for STVO from the perspective of drug-drug interaction.

\section{Background}

Schizonepeta tenuifolia (ST) is a common medicinal plant of Lamiaceae family widely spread in China, Korea and Japan. This medicinal plant has been widely used for treatment of common cold, sore throat, headache, allergic dermatitis, eczema, psoriasis and otitis media for a long time. Lines of evidence in modern pharmacological studies have demonstrated its various biological properties, such as immunomodulation [1, 2], anti-inflammation [3, 4], antioxidation [5], anti-diabetes [6]. Thus, this phytomedicine is still recorded in the present Chinese Pharmacopoeia (2020) [7].

For a long time, due to its fragrance, ST volatile oil (STVO) has been considered as the fundamental material basis of the phytomedicine and the bioactive volatile terpenoids have been listed as its quality markers, such as (+)-pulegone, $(-)$ menthone, (-)-limonene and (+)-menthofuran, which was supported by many studies [8-12]. On the other hand, as the classical dosage form of traditional Chinese medicine (TCM) for thousands of years, aqueous extract (STAE) also exhibited similar effects with ST [4, 13-15]. What's more, there were many kinds of nonvolatile components from STAE, especially the flavonoid glycosides with multiple bioactivities including hesperidin (hesperetin-7-0-rutinoside), luteoloside (luteolin 7-0glucoside) and apigetrin (apigenin 7-0-glucoside) [16-18], possibly responsible for the efficacies of ST. For instance, hesperidin could ameliorate pancreatic beta-cell dysfunction and apoptosis in streptozotocin-induced diabetic rat model and attenuate LPS-induced acute lung injury in mice by inhibiting HMGB1 release $[19,20]$. Luteoloside showed its antiinflammatory and anti-allergic activity through reducing the number of T cells, mast cells and histiocytes in mouse skin with inflammation or atopic dermatitis [21]. Apigetrin was found to inhibit LPS-induced inflammation through the inactivation of AP-1 and MAPK signaling pathways in vivo and in vitro [22]. 
In addition, in the preparation of modern Chinese medicine, ST is often extracted to yield STVO by steam distillation method. At the same time, the decoction is also obtained and considered as STAE. Then, the concentrated STAE, STVO and some pharmaceutical excipients would be mixed and prepared to produce some pharmaceutics. Many similar examples containing ST could be found in the present Chinese Pharmacopoeia (2020), such as Chuanxiong Chatiao Pills, Xiaoer Jindan Pills, Xiaoer Chiqiao Qingre Granules, Niuhuang Shangqing Pills, and so on [23]. This pharmaceutics technology not only utilizes the phytomedicine completely, but also demonstrates the two parts' effects to the full extent. Then, besides the combined effects, what influences would this combined application demonstrate on the active components in STVO and STAE as far as pharmacokinetics is concerned? To our knowledge, there have been some pharmacokinetic articles of luteoloside, apigetrin, and hesperidin in the administration form of monomeric compound, plant extract, and Chinese patent medicine [24-28]. The interaction studies of their combination with other drugs especially volatile oils were hardly involved. Therefore, when combination, whether the pharmacokinetic characteristics of the three active flavonoids would change is an interesting issue worth investigating.

In this study, in view of the effect of STVO on bioavailability of luteoloside, apigetrin, and hesperidin in STAE, a simple, rapid and sensitive UHPLC-MS method was developed for the simultaneous determination of the three bioactive flavonoids in rat plasma and applied to indicate their influence between STAE and STVO in normal rat from the perspective of pharmacokinetics. Furthermore, we hope the obtained results would be helpful for safe utilization of ST and even for understanding of volatile oil's role in combined administration of drugs.

\section{Materials And Methods}

\section{Materials and reagents}

ST was purchased from Chinese herbal medicine market in Anguo City, Hebei Province in July 2019, and then was authenticated by Prof. Qinan Wu, a botanist of Nanjing University of Chinese Medicine. The voucher specimens were kept in the laboratory.

The reference standards of luteoloside, apigetrin, hesperidin, and quercitrin (IS) were bought from Nanjing Jin Yibai Biological Technology Co., Ltd. Their purities were determined as above $98 \%$ by HPLC method. They were stored in accordance with their instructions.

Methanol and acetonitrile were both of LC-MS grade and obtained from Merck (Merck, Darmstadt, Germany). Formic acid of HPLC grade was purchased from Anaqua Chemicals Supply (ACS, Houston, USA). Ultrapure water was purified by a Milli-Q water purification system (Millipore, Burlington, MA, USA). All other reagents were of analytical grade.

\section{Preparation of STVO and STAE from ST}

Using a set of standard apparatus including a round-bottom flask, a volatile oil extractor, a condenser pipe and a heating equipment, STVO with a yield of $1.08 \%$ was obtained by a steam distillation method recorded in the present Chinese Pharmacopoeia (2020). To remove the trace water, some ahydrous sodium sulfate was added into STVO, which was then stored in the dark glass bottle at $4{ }^{\circ} \mathrm{C}$ and filtered before use. At the same time, to remove some impurities, the decoction in the round-bottom flask was transferred into a separating funnel and added with three times the amount of $95 \%$ ethanol. After the mixture was kept at $4{ }^{\circ} \mathrm{C}$ over night, the supernatants were collected and evaporated by a vacuum-rotary unit and dried in a vacuum desiccator to prepare STAE with a yield of $10.22 \%$.

\section{UHPLC-MS/MS introduction}

Chromatographic analysis was carried out on a Shimadzu Nexera X2 series UHPLC system (Shimadzu, Tokyo, Japan), equipped with a LC-30AD quaternary pump, a SIL-30AC autosampler, a DGU-20A on-line degasser, and a CT0-20AC thermostat. An Agilent Eclipse Plus $\mathrm{C}_{18}$ column $(3.0 \mathrm{~mm} \times 150 \mathrm{~mm}, 3.5 \mu \mathrm{m})$ was used for separation. The mobile phase 
consisted of with $0.2 \%$ formic acid $(A)$ and acetonitrile $(B)$ at a flow rate of $0.8 \mathrm{~mL} / \mathrm{min}$ with the gradient elution as follows: 0.0 2.0 min, 5 15\% B; $2.0 \sim 6.0 \mathrm{~min}, 15 \%$ B; 6.0 10.0 min, 15 53\% B; 10.0 12.0 min, 53 95\% B; $12.0 \sim 14.0$ min, 95 5\% B; $14.0 \sim 17.1 \mathrm{~min}, 5 \% \mathrm{~B}$. The column temperature was maintained at $30^{\circ} \mathrm{C}$ and the injection volume was $2 \mu \mathrm{L}$.

An AB Sciex 4500 Qtrap $^{T M}$ mass spectrometer (AB Sciex, Foster City, CA, USA) with electron spray ionization (ESI) in the negative mode was utilized in this study. The precursor-to-product ion transitions in multiple-reaction monitoring (MRM) mode were used for mass analysis and quantification. Some MS setting conditions were defined as follows: curtain gas pressure at $20 \mathrm{psi}$, ion spray voltage at $5000 \mathrm{~V}$, turbo gas temperature at $300{ }^{\circ} \mathrm{C}$, both nebulizer gas 1 and auxiliary gas 2 at 55 psi. Nitrogen of high purity was employed as both nebulizing gas and drying gas. All the data were obtained and analyzed by Analyst 1.6.3, a data acquisition and processing software.

\section{Animal experiments}

In the pharmacokinetic experiment, SPF male Sprague-Dawley rats (220-250 g) were supplied by Shanghai Jiesijie Experimental Animal Co., Ltd. (SCXK (Hu) 2018-0004, Shanghai, China). The rats were housed under ambient temperature of $23 \pm 2{ }^{\circ} \mathrm{C}$ with a $12 \mathrm{~h} \mathrm{light/dark}$ cycle and $50 \%$ relative humidity in the laboratory. They were well looked after in the plastic cages with fresh water and free pellet food and were acclimatized for a week. Prior to the experiment, all the rats were fasted for $12 \mathrm{~h}$ with free access to water. To minimize the pain and suffering of the rats, the experimental procedures were performed in line with the guidelines of the Animal Care and Use Committee of Nanjing University of Chinese Medicine (Nanjing, China). The protocol was also approved by the animal experimental committee of Nanjing University of Chinese Medicine (201909A008).

\section{Preparation of standard solutions and quality control samples}

The reference standards of the three target flavonoids were separately and accurately weighed and dissolved in methanol to prepare the stock standard solutions with the concentrations of $0.238 \mathrm{mg} / \mathrm{mL}$ for luteoloside, $0.193 \mathrm{mg} / \mathrm{mL}$ for apigetrin and $0.118 \mathrm{mg} / \mathrm{mL}$ for hesperidin. Then, the calibration standard solutions were obtained by mixing and serially diluting above three stock standard solutions with methanol. Additionally, IS stock solution of quercitrin was prepared with the concentration of $200 \mathrm{ng} / \mathrm{mL}$ in methanol. All these solutions were enclosed and stored at $4{ }^{\circ} \mathrm{C}$ until analysis.

The plasma samples of calibration standards were prepared by spiking $10 \mu \mathrm{L}$ of appropriate standard solution into $90 \mu \mathrm{L}$ of blank plasma to obtain the final concentrations of 1.19 238 ng/mL for luteoloside, $0.965 \sim 193 \mathrm{ng} / \mathrm{mL}$ for apigetrin, $0.590 \sim 118 \mathrm{ng} / \mathrm{mL}$ for hesperidin, respectively. Quality control (QC) samples at low, middle and high concentrations (1.19, 119 and $190 \mathrm{ng} / \mathrm{ml}$ for luteoloside; $0.965,96.5$ and $154 \mathrm{ng} / \mathrm{ml}$ for apigetrin; 0.590, 59.0 and $94.4 \mathrm{ng} / \mathrm{ml}$ for hesperidin) were also prepared according to the similar procedures.

\section{Biological sample preparation}

$100 \mu \mathrm{L}$ of plasma samples and $10 \mu \mathrm{L}$ of IS solution was added to a centrifuge tube and mixed with $500 \mu \mathrm{L}$ acetonitrile for protein precipitation. The mixture was vortexed for $3 \mathrm{~min}$ and then centrifuged at $13000 \mathrm{rpm}$ for $10 \mathrm{~min}$ at $4{ }^{\circ} \mathrm{C}$. The supernatant was transferred to another centrifuge tube and then desiccated with an Eppendorf Concentrator Plus (Hamburg, Germany). Finally, the residue was redissolved and vortex-mixed with $120 \mu \mathrm{L}$ of acetonitrile/water (50:50, v/v) for $5 \mathrm{~min}$ and centrifuged at $13000 \mathrm{rpm}$ for $10 \mathrm{~min}$. The supernatant was then injected into the UHPLC-MS/MS system for analysis.

\section{Method validation}

Based on the bioanalytical guidance of FDA, the method validation was carried out in terms of its specificity, linearity, sensitivity, accuracy, precision, matrix effect, recovery and stability. 
The chromatograms of some biological samples were compared to investigate the method specificity, including blank plasma samples, plasma samples spiked with luteoloside, apigetrin, hesperidin at lower limits of quantification (LLOQ) and IS, plasma samples after oral administration of STAE+STVO and STAE.

\section{Linearity and $L L O Q$}

The calibration curve of each analyte was obtained by plotting its peak area ratio $(y)$ to IS versus its nominal concentration (x) with weighted $\left(1 / \mathrm{x}^{2}\right)$ least-squares linear regression. The method sensitivity was evaluated by LLOQ of each analyte with its signal-to-noise ratio of 10:1 in the matrix-analyte samples, which was conformed to the lowest concentration of the standard curve with an acceptable accuracy (RE $\leq \pm 20 \%$ ) and precision (RSD $\leq 20 \%)$.

\section{Precision and accuracy}

Six QC samples at each level (low, medium, high) were used to in the method precision and accuracy test. The intra-day precision test was performed by repeatedly analyzing each QC sample in a day while the inter-day precision test was carried out by analyzing each QC sample on three consecutive days. The analytical precision was expressed by relative standard deviation (RSD) and relative error (RE), respectively. RE was calculated with this formula: $R E(\%)=$ (measuring value nominal value) $\times 100 \% /$ nominal value. The pass criterion for RSD was required to be less than $15 \%$, and that for RE was required to be within $\pm 15 \%$.

\section{Extraction recovery and matrix effect}

The extraction recovery and the matrix effect were evaluated with six replicates by the formulas as follows: $\left(A_{1} / A_{2}\right) \times 100 \%$ and $\left(A_{2} / A_{3}\right) \times 100 \%$, respectively. $A_{1}$ : the peak areas obtained from the post-treated plasma samples spiked with the target components at the three QC levels; $A_{2}$ : the peak areas obtained from the pre-treated plasma samples spiked with the target components at the three QC levels; $A_{3}$ : the peak areas obtained from the standard solutions at the equivalent concentrations. The extraction recovery and matrix effect of IS were also measured by the same approach at the identical concentration.

\section{Stability}

The stability of each analyte in rat plasma was evaluated by analyzing six replicates of the QC samples at low, middle, and high concentrations in the following practical experimental conditions: storage at $4{ }^{\circ} \mathrm{C}$ for $12 \mathrm{~h}$, three freeze-thaw cycles (freezing at $-80{ }^{\circ} \mathrm{C}$ for $24 \mathrm{~h}$ and thawing at room temperature) and long-term storage at $-80{ }^{\circ} \mathrm{C}$ for 2 weeks. The acceptable $\mathrm{RE}$ was within $\pm 15.0 \%$ of the nominal concentration.

\section{Pharmacokinetic study}

For pharmacokinetic study, all the rats were divided into two groups ( $\mathrm{n}=6$ per group) randomly. The given dosages of two test extracts were equivalent to about $5 \mathrm{~g} \mathrm{ST} / \mathrm{kg}$. STAE group: the rats were administrated orally with STAE $(500 \mathrm{mg} / \mathrm{kg})$, dissolved in $0.5 \%$ carboxy methyl cellulose sodium (CMC-Na) solution. STAE+STVO group: the rats were administrated orally with STAE $(500 \mathrm{mg} / \mathrm{kg})$ and STVO $(50 \mathrm{mg} / \mathrm{kg})$, dissolved in $0.5 \% \mathrm{CMC}-\mathrm{Na}$ solution. Blood samples were collected from the venous plexus of the eye socket and transferred in a heparinized centrifuge tube $(1.5 \mathrm{~mL})$ after oral administration $(0,5,10,20,30,45,60,120,240,480$, and $720 \mathrm{~min})$. Each sample was immediately centrifuged at $13000 \mathrm{rpm}$ for $10 \mathrm{~min}$ at $4{ }^{\circ} \mathrm{C}$ to acquire the plasma. At last, the plasma was immediately stored at $-80{ }^{\circ} \mathrm{C}$ until analysis.

\section{Data analysis}

The plasma concentrations of the three flavonoids during the experiment were calculated according to the daily calibration curve. With Drug And Statistics Version 3.2.8 (DAS 3.2.8) software (Mathematical Pharmacology Professional Committee 
of China, Shanghai, China), a non-compartmental method was employed to analyze some representative pharmacokinetic parameters, such as the time to reach maximum concentration $\left(T_{\text {max }}\right)$, elimination half-life time $\left(T_{1 / 2}\right)$, the area under the concentration curves $\left(\mathrm{AUC}_{0 \rightarrow \mathrm{t}}\right.$ and $\left.\mathrm{AUC}_{0 \rightarrow \infty}\right)$ and the maximum concentration $\left(\mathrm{C}_{\mathrm{max}}\right)$. Data were demonstrated as the mean \pm standard deviation (SD) with triplicate. The differences of pharmacokinetic parameters from two groups were tested by Student's t-test. Statistical analysis was operated by Graphpad Prism 7.0 software.

\section{Results And Methods}

\section{Optimization of chromatographic and MS conditions}

As far as peak shape and analysis time were concerned, it was revealed upon comparison that an Agilent Eclipse Plus $\mathrm{C}_{18}$ column $(3.0 \mathrm{~mm} \times 150 \mathrm{~mm}, 3.5 \mu \mathrm{m})$ showed better performance than a Phenomenon Luna $\mathrm{C}_{18}$ column $(4.6 \mathrm{~mm} \times 250 \mathrm{~mm}, 5$ $\mu \mathrm{m})$, a Hanbon Megres $\mathrm{C}_{18}$ column $(4.6 \mathrm{~mm} \times 250 \mathrm{~mm}, 5 \mu \mathrm{m})$. The mobile phase system consisting of $0.2 \%$ formic acid and acetonitrile was employed for gradient elution with the flow rate of $0.8 \mathrm{~mL} / \mathrm{min}$.

Due to similar chemical structure, appropriate retention time and nonexistence in ST and rats, quercitrin was selected as the internal standard for quantification of luteoloside, apigetrin, and hesperidin (Figure 1). For the responses of these four flavonoid glycosides, the negative mode was much better in comparison with the positive mode during MS detection. So, their deprotonated ions $[\mathrm{M}-\mathrm{H}]^{-}$were considered as the precursor ions (Q1) and the products of glycosidic bond cleavages were selected as the product ions (Q3). Compound dependent MS parameters including collision energy (CE), collision cell exit potential (CXP) and declustering potential (DP) values were optimized and designed by Applied Biosystems/MDS Sciex Analyst software 1.5.2. These parameters were demonstrated in Table 1.

\section{Sample preparation}

It is well known that protein precipitation, liquid-liquid extraction and solid phase extraction are the common methods for plasma sample preparation. In our trial test, low extraction recoveries of the analytes were found in liquid-liquid extraction and could not meet the specifications of pharmaceutical analysis. Too many cartridges would be used and significant time would be required when solid phase extraction was employed. Thus, protein precipitation with acetonitrile was selected to prepare plasma samples owing to its easy operation, high efficiency and low cost.

\section{Method validation}

\section{Specificity}

The representative chromatograms acquired from blank plasma, blank plasma added with the three target flavonoids at LLOQ and IS, and plasma sample at $1 \mathrm{~h}$ after oral administration of STAE and STAE+STVO are shown in Figure 2. Due to the efficient sample preparation and high selectivity of MRM, there was no obvious endogenous interference for the separation and analysis of the four flavonoids in the plasma.

\section{Linearity and $L L O Q$}

The linear regression equations, correlation coefficients $(r)$ and LLOQs of luteoloside, apigetrin and hesperidin were summarized in Table 2 . The correlation coefficients $(\geq 0.9995)$ indicated their good linearities and the LLOQs $(0.590 \otimes 1.19$ $\mathrm{ng} / \mathrm{mL}$ ) were appropriate for quantitative detection.

\section{Precision and accuracy}

As shown in Table 3, the results of the intra- and inter-day precision and accuracy of the analytes in QC samples were exhibited. For the three QC levels of the analytes, in the intra-day test, RSD values varied from $3.47 \%$ to $10.45 \%$, while RE 
values ranged between $-3.82 \%$ and $5.43 \%$. Similarly, in the inter-day test, RSD values varied from $4.29 \%$ to $11.28 \%$, while RE values ranged between $-7.09 \%$ and $9.24 \%$. All these precision and accuracy results were within the acceptable ranges for analysis in biological media, indicating the reliability and reproducibility of the method.

\section{Extraction recovery and matrix effect}

As for the three QC levels, the average extraction recoveries of luteoloside, apigetrin and hesperidin ranged from $77.41 \%$ to $109.79 \%$ while their average matrix effects were between $83.41 \%$ and $112.67 \%$ (Table 4). Besides, all of the RSD values were below $15.00 \%$. Thus, the results within the acceptable ranges demonstrated that the analytes could be extracted from the plasma efficiently and matrix effects were seldom found for this method.

\section{Stability}

The stability of the three analytes in rat plasma during the sample storing and processing procedures was fully evaluated. The results (Table 5) showed that luteoloside, apigetrin and hesperidin were stable in rat plasma after three freeze-thaw cycles, for $12 \mathrm{~h}$ at $4{ }^{\circ} \mathrm{C}$ and for 2 weeks at $-80^{\circ} \mathrm{C}$ after plasma sample preparation. The RE values were calculated in the range from $-7.13 \%$ to $11.54 \%$ and the RSD values were not more than $9.38 \%$, which demonstrated that the sample solutions were stable under above various conditions.

\section{Pharmacokinetic study}

The developed assay method was sensitive and accurate enough to be applied in the pharmacokinetic study of luteoloside, apigetrin, and hesperidin in rat plasma following oral administration of STAE and STAE+STVO. The plasma concentrationtime curves of the three analytes are present in Figure 3. The pharmacokinetic parameters are summarized in Table 6, including $\mathrm{T}_{\max }, \mathrm{T}_{1 / 2}, \mathrm{C}_{\max }, \mathrm{AUC}_{0 \rightarrow \mathrm{t}}$ and $\mathrm{AUC}_{0 \rightarrow \infty}$.

In Figure 3, it can be seen intuitively that the concentration-time curve of each flavonoid glycoside in rat plasma after oral administration of STAE+STVO is similar as that of STAE. Acturally, the pharmacokinetic profiles of each analyte in the two groups differentiated to some extent. The results demonstrated that the mean $T_{1 / 2}$ values of luteoloside, apigetrin, and hesperidin in STAE+STVO group were found 1.12, 1.09, and 1.13 fold, respectively, compared to those in STAE group. However, there were no notable differences observed, indicating some weak influence on elimination procedure of the three analytes. In addition, for the three flavonoids, after co-administration of STAE and STVO, the values of $A_{0 \rightarrow t}$ and $A U C_{0 \rightarrow \infty}$ were investigated significantly larger than administration of STAE alone $(p<0.01, p<0.05)$, except AUC $_{0 \rightarrow \infty}$ value of apigetrin. The data reminded us promoting effect of STVO on absorption of the three analytes. For hesperidin and apigetrin, $T_{\max }$ values in STAE+STVO group were markedly longer than those in STAE group $(p<0.01)$ while $\mathrm{C}_{\max }$ values were increased without notable differences. On the contrary, $C_{\max }$ value of luteoloside was elevated notably $(p<0.05)$ while no significant difference was found for its $T_{\max }$ value as far as the same comparison was concerned. It was concluded that STVO might ameliorate absorption and improve bioavailability of the three analytes.

\section{Discussion}

In the past decades, it has become a consensus that the plant-originated volatile oils are investigated as the favourable transdermal absorption enhancing agents, such as the volatile oils from Rosmarinus officinalis [29], Magnolia fargesii [30], Eugenia caryophyllata [31] and Alpiniae Officinarum [32]. Moreover, it has been documented that the volatile oil from Acori Tatarinowii might have promoting effect on pharmacokinetic fates of three coumarins including byakangelicin, xanthotoxol and oxypeucedanin hydrate from Angelicae Dahuricae when co-administration in rat [33]. In another study, the volatile oil from Xiangfu Siwu Decoction (a famous formula of traditional Chinese medicine) has been found to improve the absorption and bioavailability of ten non-volatile components (caffeic acid, ferulic acid, gallic acid, vanillic acid, albiflorin, paeoniflorin, berberine, protopine, tetrahydrocolumbamine and tetrahydropalmatine) in the same formula [34]. Taken 
together, these findings indicated that the volatile oils could enhance the absorption and bioavailability of some drugs not only through transdermal absorption, but also through intestinal absorption, which was consistent with the results of our present study.

Our investigation verified the rationality of oral co-administration of STAE and STVO in terms of pharmacokinetics, which would be helpful for the pharmaceutical technology study of the Chinese herbal preparation containing ST and for the novel application exploration of STVO. However, there still existed some interesting issues. Except for the pharmacokinetic parameters, would the metabolites of the three analytes in STAE be affected by STVO? Which volatile component(s) showed absorption-promoting effect? What was the absorption-promoting mechanism of STVO? Was P-glycoprotein involved? Which effects would STAE show on the pharmacokinetic profiles of the components in STVO after oral coadministration? These would be listed as the research priorities in the future.

\section{Conclusions}

In the present study, a sensitive and rapid UHPLC-MS/MS analytical method was firstly established for simultaneous quantitative analysis of luteoloside, apigetrin, and hesperidin in rat plasma. This method provided eligible recovery and stability with fine precision and accuracy and then was successfully applied to the pharmacokinetic study of three bioactive flavonoid glycisodes after oral administration of STAE and STAE + STVO. The results obtained from this study demonstrated that STVO could promote the absorption and bioavailability of the three flavonoid glycosides in vivo. Therefore, we hope that these findings would help to broaden the applied range of STVO and to provide some scientific basis and guidance for the safe clinical use of ST.

\section{Abbreviations}

ST, Schizonepeta tenuifolia; STVO, Schizonepeta tenuifolia volatile oil; STAE, Schizonepeta tenuifolia aqueous extract; UHPLC-MS/MS, ultra high performance liquid chromatography-tandem mass spectrometry

\section{Declarations}

\section{Acknowledgements}

The authors wish to show their gratitude to Department of Pharmacy, Children's Hospital of Nanjing Medical University and Jiangsu Collaborative Innovation Center of Chinese Medicinal Resources Industrialization, Nanjing University of Chinese Medicine.

\section{Conflicts of interest}

The authors declare no conflict of interest.

\section{Fundings}

This study was financially supported by National Natural Science Foundation of China $(81473313,81973435)$, Special Key Projects of Basic Scientific and Technological Work of National Ministry of Science and Technology (2014FY111), Topnotch Academic Programs Project of Jiangsu Higher Education Institutions (TAPP-PPZY2015A070), 333 High-level Talents Training Project Funded by Jiangsu Province (BRA2020071).

\section{Authors' contributions}

MQS and SY designed the experiments; STL, YLJ, and YDC carried out the experiments; STL, BHB and FFC performed the data analysis; LZ and QNW provided the fund; STL wrote the original draft; MQS and PDC revised the manuscript. All 
authors have read and approved the final manuscript.

\section{Availability of data and materials}

All data in this article are available from the corresponding author upon request.

\section{Ethics approval and consent to participate}

The experiment concerning animals was approved by the animal experimental committee of Nanjing University of Chinese Medicine (201909A008) and was carried out according to the guidelines of the Animal Care and Use Committee of Nanjing University of Chinese Medicine.

\section{Consent for publication}

All authors have provided consent for publication in Chinese Medicine.

\section{References}

1. Choi YY, Kim MH, Kim JH, Jung HS, Sohn Y, Choi YJ, et al. Schizonepeta tenuifolia inhibits the development of atopic dermatitis in mice. Phytother Res. 2012;27(8):1131-5.

2. Lin YH, Chen HY, Chiu JC, Chen KJ, Ho HY, Yang SH. Immunomodulation effects of Schizonepeta tenuifolia Briq. on the IgE-induced allergic model of RBL-2H3 cells. Evid Complement Alternat Med. 2018. https://doi.org/10.1155/2018/6514705.

3. Byun MW. Schizonepeta tenuifolia ethanol extract exerts anti-inflammatory activity through the inhibition of TLR4 signaling in lipopolysaccharide-stimulated macrophage cells. J Med Food. 2014;17(3):350-6.

4. Kim SJ, Kim JS, Choi IY, Kim DH, Kim MC, An HJ, et al. Anti-inflammatory activity of Schizonepeta tenuifolia through the inhibition of MAPK phosphorylation in mouse peritoneal macrophages. Am J Chinese Med. 2008;36(6):1145-58.

5. Tae KS, Kim SJ. Inhibition of iNOS and DNA oxidation by methanol extract of Schizonepeta tenuifolia. Trop J Pharm Res. 2012;11(3):397-404.

6. Do MH, Choi J, Kim Y, Park HY, Ha SK, Hui JY. Schizonepeta tenuifolia reduces methylglyoxal-induced cytotoxicity and oxidative stress in mesangial cells. J Function Food. 2019. https://doi.org/10.1016/j.jff.2019.103531.

7. Chinese Pharmacopoeia Commission. Pharmacopoeia of the People's Republic of China. Vol. I. Beijing: China Medical Science and Technology Press; 2020. pp. 243-4. (In Chinese).

8. He T, Tang Q, Zeng N, Gou L, Liu JW, Yang J, et al. Study on effect and mechanism of volatile oil of Schizonepetae herba and its essential components against influenza virus. China J Chin Mater Med. 2013;38(11):1772-7. (In Chinese).

9. Lyu HJ, Wen TQ, Luo J, Liu XB, Yang J, Zeng N. Study on mechanism of essential oils of Schizonepeta tenuifolia Briq. in endotoxin poisoning mice via NLRP3 inflammasome pathway. Chin Pharmacol Bull. 2019;35(3):371-5. (In Chinese).

10. Zang LQ, Hu F, Wei M, Wang NP, Wei JB. Research on anti-tumor effect of essential oil in Schizonepeta tenuifolia Briq. and its inducing apoptosis. Guangxi J Chin Tradit Med. 2006;29(4):246-8. (In Chinese).

11. Zeng N, Yang X, Zhao L, Liu XS, Zhou ZH, Song MF. The anti-inflammatory effect of herba Schizonepetae volatile oil on carrageenan-induced acute pleurisy model in rats. Pharmacol Clin Chin Materia Medica. 2010;1(1):31-3. (In Chinese).

12. Yu S, Chen YW, Zhang L, Shan MQ, Tang YP, Ding AW. Quantitative comparative analysis of the bio-active and toxic constituents of leaves and spikes of Schizonepeta tenuifolia at different harvesting times. Int J Mol Sci. 2011;12(10):6635-44.

13. Kang H, Han SW, Hong JW, Sohn NW. Suppression of tumour necrosis factor-alpha by Schizonepeta tenuifolia water extract via inhibition of I kappa B alpha degradation and Jun N-terminal kinase/stress-activated protein kinase 
activation. J Pharm Pharmacol. 2010;62(8):1069-76.

14. Wang BS, Huang GJ, Tai HM, Huang MH. Antioxidant and anti-inflammatory activities of aqueous extracts of Schizonepeta tenuifolia Briq. Food Chem Toxicol. 2012;50(3-4):526-31.

15. Shin TY, Jeong HJ, Jun SM, Chae HJ, Kim HR, Baek SH, et al. Effect of Schizonepeta tenuifolia extract on mast cellmediated immediate-type hypersensitivity in rats. Immunopharm Immunot. 1999;21(4):705-15.

16. Huang XH, Chen J, Xu XQ, Zhang WT, Zhao CC. A new phenolic compound from Schizonepeta tenuifolia. Chem Nat Compd. 2016;52(6):1005-7.

17. Kubo M, Sasaki H, Endo T, Taguchi H, Yosioka I. The constituents of Schizonepeta tenuifolia Briq. Il.: structure of a new monoterpene glucoside, schizonepetoside C. Chem Pharm Bull. 1986;34(8):3097-101.

18. Lee IK, Kim MA, Lee SY, Lee JH, Lee KR. Phytochemical constituents of Schizonepeta tenuifolia Briquet. Nat Prod Sci. 2008;14(2):100-6.

19. Hanchang W, Khamchan A, Wongmanee N, Seedadee C. Hesperidin ameliorates pancreatic beta-cell dysfunction and apoptosis in streptozotocin-induced diabetic rat model. Life Sci. 2019. https://doi.org/2019.10.1016/j.Ifs.2019.116858.

20. Liu XX, Yu DD, Chen MJ, Sun T, Li G, Huang WJ, et al. Hesperidin ameliorates lipopolysaccharide-induced acute lung injury in mice by inhibiting HMGB1 release. Int Immunopharmacol. 2015;25(2):370-6.

21. Szekalska M, Sosnowska K, Tomczykowa M, Winnicka K, Kasacka I, Tomczyk M. In vivo anti-inflammatory and antiallergic activities of cynaroside evaluated by using hydrogel formulations. Biomed Pharmacother. 2020. https://doi.org/10.1016/j.biopha.2019.109681.

22. Hu WC, Wang XF, Wu L, Shen T, Ji LL, Zhao XH, et al. Apigenin-7-O-beta-D-glucuronide inhibits LPS-induced inflammation through the inactivation of AP-1 and MAPK signaling pathways in RAW 264.7 macrophages and protects mice against endotoxin shock. Food Funct. 2016;7(2):1002-13.

23. Chinese Pharmacopoeia Commission. Pharmacopoeia of the People's Republic of China. Vol. I. Beijing: China Medical Science and Technology Press; 2020. pp. 517-8. 535, 553-554, 642-643 (In Chinese).

24. Gu YY, Zhang X, Xie M, Tao XJ, Zhang WJ, Zhang CG, et al. Pharmacokinetics of hesperidin and its hepatic, gastric and intestinal first-pass effects in rats. Lat Am J Pharm. 2019;38(8):1498-504.

25. Qiu F, Li ZX, He L, Wang D. HPLC-ESI-MS. /MS analysis and pharmacokinetics of luteoloside, a potential anticarcinogenic component isolated from Lonicera japonica, in beagle dogs. Biomed Chromatogr. 2013;27(3):311-7.

26. Chen ZX, Ying XX, Meng S, Zhu X, Jiang H, Cao QS, et al. LC determination of luteolin-7-O-beta-D-glucoside and apigenin-7-O-beta-D-glucoside in rat plasma after administration of Humulus scandens extract and its application to pharmacokinetic studies. Nat Prod Res. 2012;26(6):530-9.

27. Chen ZX, Ying XX, Meng S, Zhu X, Jiang H, Cao QS, et al. High-performance liquid chromatographic determination and pharmacokinetic study of apigenin-7-0-beta-D-glucoside in rat plasma after intravenous administration. Arch Pharm Res. 2011;34(5):741-6.

28. Zhu HY, Guan J, Shi JY, Pan X, Chang S, Zhang TY, et al. Simultaneous determination of eight bioactive constituents of Zhi-Zi-Hou-Po decoction in rat plasma by ultra high performance liquid chromatography with tandem mass spectrometry and its application to a pharmacokinetic study. J Sep Sci. 2020;43(2):406-17.

29. Akbari J, Saeedi M, Farzin D, Morteza-Semnani K, Esmaili Z. Transdermal absorption enhancing effect of the essential oil of Rosmarinus officinalis on percutaneous absorption of Na diclofenac from topical gel. Pharm Biol. 2015;53(10):1442-7.

30. Fang JY, Tsai TH, Hung CF, Wong WW. Development and evaluation of the essential oil from Magnolia fargesii for enhancing the transdermal absorption of theophylline and cianidanol. J Pharm Pharmacol. 2004;56(12):1493-500.

31. Shen Q, Li W, Li W. The effect of clove oil on the transdermal delivery of ibuprofen in the rabbit by in vitro and in vivo methods. Drug Dev Ind Pharm. 2007;33(12):1369-74. 
32. Dong J, Zhu XM, Wu FY, Yang BQ, Feng H, Dong YF, et al. Development of galangal essential oil-based microemulsion gel for transdermal delivery of flurbiprofen: simultaneous permeability evaluation of flurbiprofen and 1,8-cineole. Drug Dev Ind Pharm. 2020;46(1):91-100.

33. Shi BM, Liu JH, Zhang Q, Wang SX, Jia P, Bian LJ, et al. Effect of co-administration of Acori Tatarinowii rhizoma volatile oil on pharmacokinetic fate of xanthotoxol, oxypeucedanin hydrate, and byakangelicin from Angelicae Dahuricae Radix in rat. J Sep Sci. 2020;43(12):2349-62.

34. Pan Y, Qian DW, Liu P, Zhang Y, Zhu ZH, Zhang L, et al. The influence of essential oils from Xiang-Fu-Si-Wu Decoction on its nonvolatile components and its application for pharmacokinetics in normal rats. J Chromatogr $B$.

2017;1060:221-30.

\section{Tables}

Table 1 MS parameters of luteoloside, apigetrin, hesperidin and quercitrin (IS)

\begin{tabular}{|lllllll|}
\hline Compound & $\mathrm{t}_{\mathrm{R}}(\mathrm{min})$ & $\mathrm{Q} 1(\mathrm{~m} / \mathrm{z})$ & $\mathrm{Q} 3(\mathrm{~m} / \mathrm{z})$ & $\mathrm{DP}(\mathrm{V})$ & $\mathrm{CXP}(\mathrm{eV})$ & $\mathrm{CE}(\mathrm{eV})$ \\
\hline Luteoloside & 7.86 & 447.1 & 285.0 & 210 & 25 & 36 \\
\hline Quercitrin $(\mathrm{IS})$ & 8.54 & 447.2 & 301.0 & 180 & 15 & 30 \\
\hline Apigetrin & 10.19 & 431.1 & 269.0 & 185 & 19 & 34 \\
Hesperidin & 12.22 & 609.2 & 301.0 & 170 & 21 & 32 \\
\hline
\end{tabular}

Table 2 The regression equations, linear ranges, correlation coefficients and LLOQs of luteoloside, apigetrin and hesperidin

\begin{tabular}{|lllll|}
\hline Analyte & Regression equation & Linear range $(\mathrm{ng} / \mathrm{mL})$ & $r$ & LLOQ $(\mathrm{ng} / \mathrm{mL})$ \\
\hline Luteoloside & $\mathrm{y}=0.0750 \mathrm{x}+0.1836$ & $1.19 \varangle 238$ & 0.9998 & 1.19 \\
\hline Apigetrin & $\mathrm{y}=0.0598 \mathrm{x}-0.1277$ & $0.965 \bigotimes 193$ & 0.9995 & 0.965 \\
\hline Hesperidin & $\mathrm{y}=0.0711 \mathrm{x}+0.2943$ & $0.590 \otimes 118$ & 0.9996 & 0.590 \\
\hline
\end{tabular}

Table 3 Intra-day and inter-day precision and accuracy of luteoloside, apigetrin and hesperidin 


\begin{tabular}{|c|c|c|c|c|c|}
\hline \multirow[t]{2}{*}{ Analyte } & \multirow{2}{*}{$\begin{array}{l}\text { Concentration } \\
(\mathrm{ng} / \mathrm{mL})\end{array}$} & \multicolumn{2}{|l|}{ Intra-day } & \multicolumn{2}{|l|}{ Inter-day } \\
\hline & & Precision (RSD, \%) & Accuracy (RE, \%) & Precision (RSD, \%) & Accuracy (RE, \%) \\
\hline \multirow[t]{3}{*}{ Luteoloside } & 1.19 & 10.45 & -1.47 & 8.86 & -0.37 \\
\hline & 119 & 5.69 & 1.43 & 5.71 & 3.63 \\
\hline & 190 & 3.47 & -1.29 & 4.29 & -7.09 \\
\hline \multirow[t]{3}{*}{ Apigetrin } & 0.965 & 9.52 & 3.85 & 11.28 & 7.14 \\
\hline & 96.5 & 8.95 & -3.82 & 9.69 & 2.54 \\
\hline & 154 & 5.53 & 0.91 & 4.53 & 1.90 \\
\hline \multirow[t]{3}{*}{ Hesperidin } & 0.590 & 9.28 & 5.43 & 7.46 & 9.24 \\
\hline & 59.0 & 6.03 & 1.36 & 7.62 & 4.81 \\
\hline & 94.4 & 5.77 & -1.34 & 5.08 & -1.00 \\
\hline
\end{tabular}

Table 4 Recoveries and matrix effects of luteoloside, apigetrin and hesperidin

\begin{tabular}{|llllll|}
\hline Analyte & Concentration $(\mathrm{ng} / \mathrm{mL})$ & Recovery $(\%)$ & RSD (\%) & Matrix effect (\%) & RSD (\%) \\
\hline Luteoloside & 1.19 & $80.34 \pm 3.23$ & 4.02 & $88.40 \pm 2.63$ & 2.72 \\
\cline { 2 - 6 } & 119 & $89.48 \pm 1.89$ & 2.11 & $84.48 \pm 2.45$ & 7.48 \\
\cline { 2 - 6 } Apigetrin & 190 & $91.72 \pm 5.44$ & 5.93 & $101.56 \pm 4.04$ & 3.01 \\
& 0.965 & $77.41 \pm 6.28$ & 8.11 & $97.01 \pm 3.85$ & 9.53 \\
& 96.5 & $82.67 \pm 4.43$ & 5.36 & $112.67 \pm 9.53$ & 6.31 \\
\hline Hesperidin & 0.590 & $94.6 \pm 6.93$ & 7.33 & $96.9 \pm 4.73$ & 4.70 \\
\cline { 2 - 6 } & 59.0 & $88.34 \pm 7.95$ & 9.00 & $83.41 \pm 9.15$ & 8.86 \\
& 94.4 & $109.79 \pm 8.64$ & 7.87 & $97.93 \pm 4.46$ & 3.51 \\
\cline { 2 - 6 } & & $97.83 \pm 6.49$ & 6.63 & $107.38 \pm 7.29$ & 4.82 \\
\hline
\end{tabular}

Table 5 Stability of luteoloside, apigetrin and hesperidin 


\begin{tabular}{|c|c|c|c|c|c|c|c|}
\hline \multirow[t]{2}{*}{ Analyte } & \multirow{2}{*}{$\begin{array}{l}\text { Concentration } \\
(\mathrm{ng} / \mathrm{mL})\end{array}$} & \multicolumn{2}{|c|}{$4^{\circ} \mathrm{C}$ for $12 \mathrm{~h}$} & \multicolumn{2}{|c|}{3 freeze-thaw cycles } & \multicolumn{2}{|c|}{$-80^{\circ} \mathrm{C}$ for 2 weeks } \\
\hline & & RSD (\%) & RE (\%) & RSD (\%) & $\mathrm{RE}(\%)$ & RSD (\%) & RE (\%) \\
\hline \multirow[t]{3}{*}{ Luteoloside } & 1.19 & 8.56 & 7.35 & 9.38 & 5.15 & 7.61 & 1.47 \\
\hline & 119 & 6.56 & 6.69 & 6.23 & 0.70 & 4.27 & 0.54 \\
\hline & 190 & 4.83 & -5.62 & 4.81 & -7.13 & 4.60 & -5.20 \\
\hline \multirow[t]{3}{*}{ Apigetrin } & 0.965 & 4.93 & 11.54 & 4.62 & -4.95 & 6.15 & 7.14 \\
\hline & 96.5 & 4.65 & 6.87 & 3.70 & 1.60 & 6.10 & 1.37 \\
\hline & 154 & 4.43 & 5.96 & 3.92 & -1.34 & 5.06 & -2.22 \\
\hline \multirow[t]{3}{*}{ Hesperidin } & 0.590 & 8.29 & 4.89 & 7.18 & -1.63 & 7.47 & -5.43 \\
\hline & 59.0 & 7.24 & 9.48 & 6.33 & 7.44 & 4.40 & 4.30 \\
\hline & 94.4 & 4.20 & 8.72 & 4.96 & -1.60 & 4.68 & -1.00 \\
\hline
\end{tabular}

Table 6 Pharmacokinetic parameters of luteoloside, apigetrin and hesperidin after oral administration of STAE and STAE+STVO

\begin{tabular}{|c|c|c|c|c|c|c|}
\hline Analyte & Group & $\mathrm{T}_{\max }(\mathrm{h})$ & $\mathrm{C}_{\max }(\mathrm{ng} / \mathrm{mL})$ & $\mathrm{T}_{1 / 2}(\mathrm{~h})$ & $\begin{array}{l}\mathrm{AUC}_{0 \rightarrow \mathrm{t}} \\
(\mathrm{h} \cdot \mathrm{mg} / \mathrm{L})\end{array}$ & $\mathrm{AUC}_{0 \rightarrow \infty}(\mathrm{h} \cdot \mathrm{mg} / \mathrm{L})$ \\
\hline \multirow[t]{2}{*}{ Luteoloside } & STAE & $0.667 \pm 0.258$ & $16.552 \pm 2.421$ & $4.000 \pm 1.237$ & $40.476 \pm 2.905$ & $45.064 \pm 5.241$ \\
\hline & STAE+STVO & $0.958 \pm 0.102$ & $22.913 \pm 6.192^{\star}$ & $4.359 \pm 1.988$ & $57.934 \pm 5.881^{\star \star}$ & $64.988 \pm 8.724^{\star \star}$ \\
\hline \multirow[t]{2}{*}{ Apigetrin } & STAE & $0.444 \pm 0.086$ & $12.283 \pm 2.233$ & $8.646 \pm 1.901$ & $25.772 \pm 1.369$ & $39.394 \pm 4.607$ \\
\hline & STAE+STVO & $0.750 \pm 0.000 * *$ & $13.506 \pm 2.558$ & $9.767 \pm 3.045$ & $31.479 \pm 1.365^{\star \star}$ & $53.743 \pm 10.829$ \\
\hline \multirow[t]{2}{*}{ Hesperidin } & STAE & $0.514 \pm 0.134$ & $23.882 \pm 4.431$ & $5.182 \pm 1.231$ & $31.139 \pm 1.740$ & $42.137 \pm 9.395$ \\
\hline & STAE+STVO & $0.875 \pm 0.137 * *$ & $31.215 \pm 5.354$ & $5.797 \pm 0.935$ & $55.439 \pm 3.013^{\star *}$ & $60.206 \pm 3.672^{*}$ \\
\hline
\end{tabular}

** $p<0.01,{ }^{*} p<0.05$ versus STAE group

\section{Figures}


<smiles>O=c1cc(-c2ccc(O)c(O)c2)oc2cc(O[C@@H]3O[C@H](CO)[C@@H](O)[C@H](O)[C@H]3O)cc(O)c12</smiles>

(1)<smiles>COc1ccc([C@H]2CC(=O)c3c(O)cc(O[C@@H]4O[C@H](CO[C@@H]5O[C@@H](C)[C@H](O)[C@H](O)[C@H]5O)[C@@H](O)[C@H](O)[C@H]4O)cc3O2)cc1O</smiles>

(3)<smiles>O=c1cc(-c2ccc(O)cc2)oc2cc(O[C@@H]3O[C@H](CO)[C@@H](O)[C@H](O)[C@H]3O)cc(O)c12</smiles>

(2)

(4)

\section{Figure 1}

Chemical structures of luteoloside (1), apigetrin (2), hesperidin (3) and quercitrin (4, IS)<smiles>O=c1cc(-c2ccc(O)c(O)c2)oc2cc(O[C@@H]3O[C@H](CO)[C@@H](O)[C@H](O)[C@H]3O)cc(O)c12</smiles>

(1)<smiles>COc1ccc([C@H]2CC(=O)c3c(O)cc(O[C@@H]4O[C@H](CO[C@@H]5O[C@@H](C)[C@H](O)[C@H](O)[C@H]5O)[C@@H](O)[C@H](O)[C@H]4O)cc3O2)cc1O</smiles>

(3)<smiles>O=c1cc(-c2ccc(O)cc2)oc2cc(O[C@@H]3O[C@H](CO)[C@@H](O)[C@H](O)[C@H]3O)cc(O)c12</smiles>

(2)<smiles>C[C@@H]1O[C@H](Oc2c(-c3ccc(O)c(O)c3)oc3cc(O)cc(O)c3c2=O)[C@H](O)[C@H](O)[C@H]1O</smiles>

(4)

Figure 1 
Chemical structures of luteoloside (1), apigetrin (2), hesperidin (3) and quercitrin (4, IS)

(1)

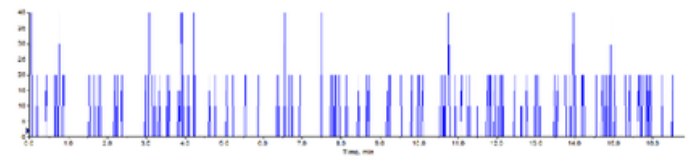

(1)
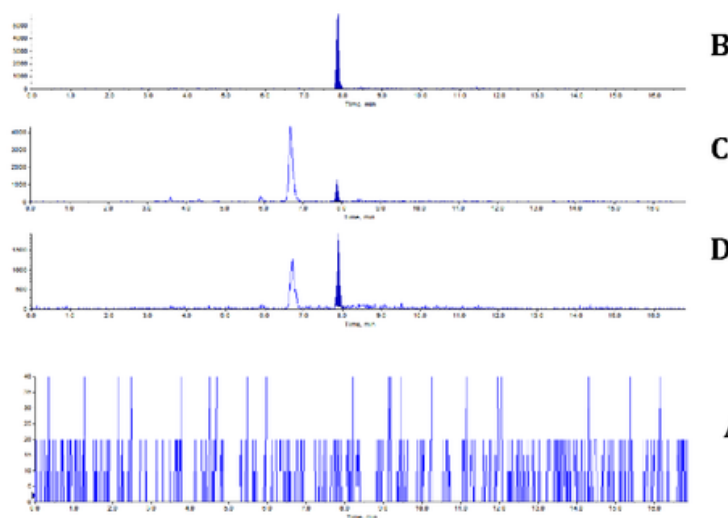

(3)

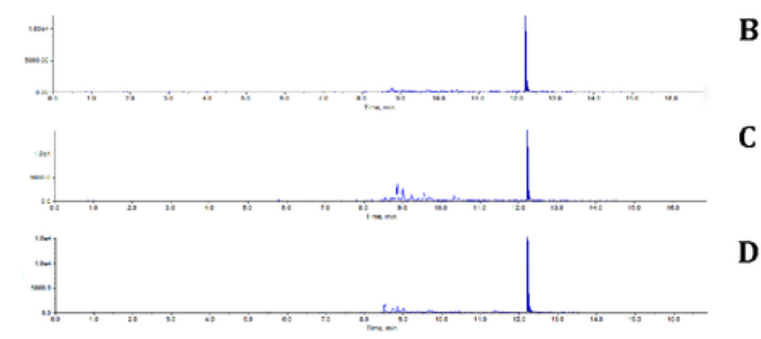

A

B

D
(2)

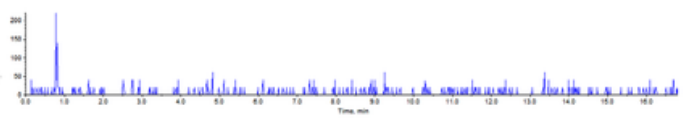

A

B
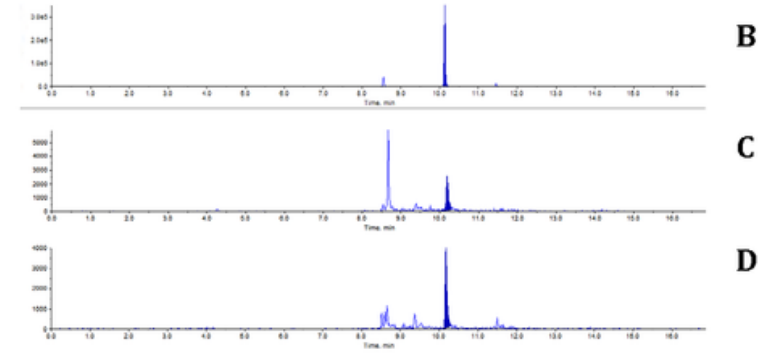

D

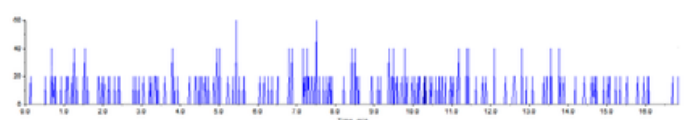

(4)

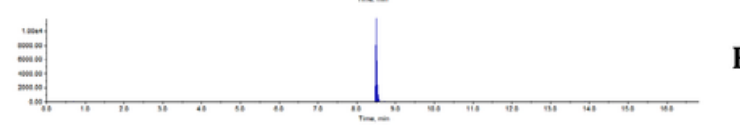

C

D

A

B

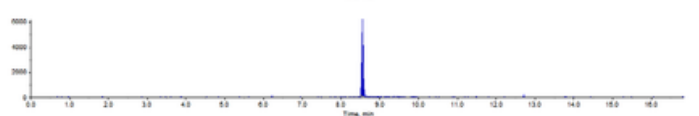

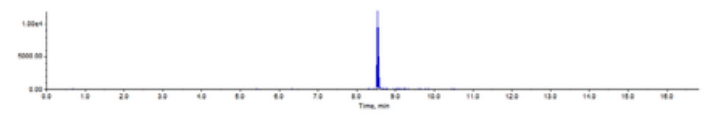

Figure 2

Representative MRM chromatograms of luteoloside (1), apigetrin (2), hesperidin (3) and quercitrin (4, IS) in rat plasma (A. blank plasma; B. blank plasma added with the three analytes at LLOQ and IS; C. plasma sample at $1 \mathrm{~h}$ after oral administration of STAE; D. plasma sample at $1 \mathrm{~h}$ after oral administration of STAE and STVO) 
(1)
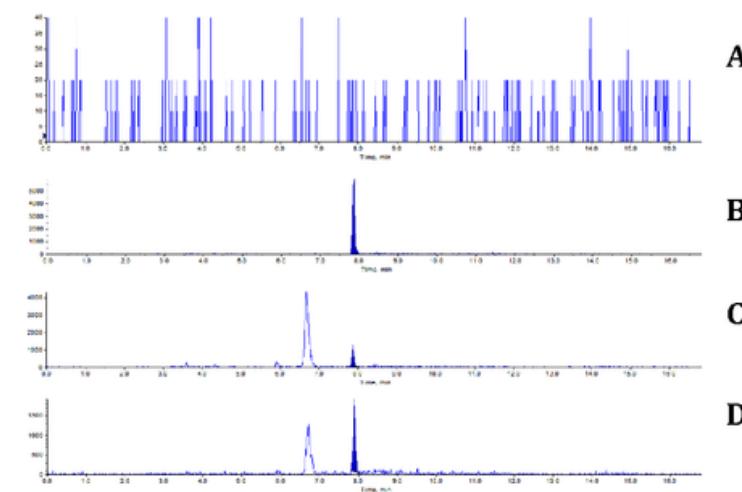

D

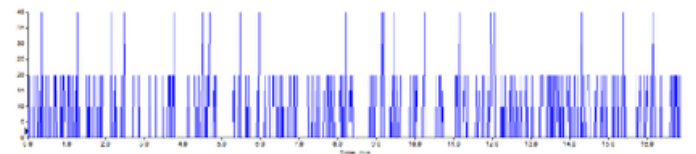

(3)

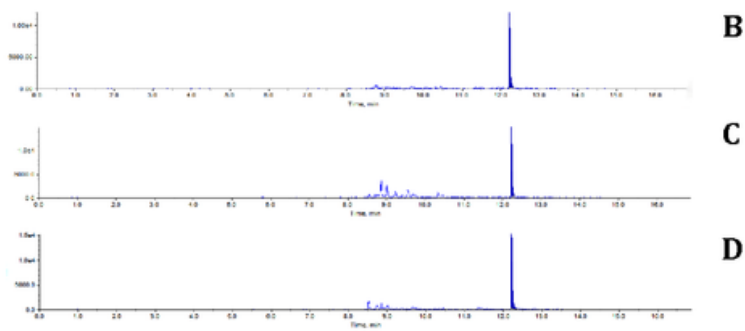

(2)

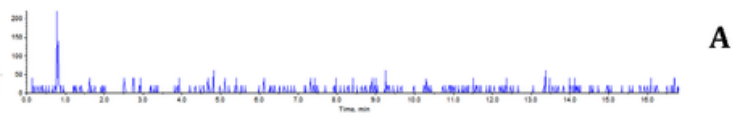

B

C

D

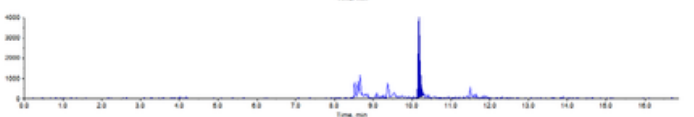

A

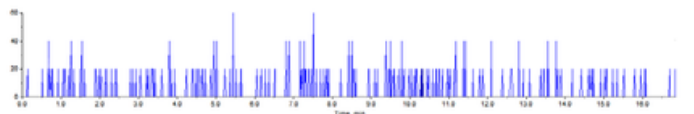

(4)
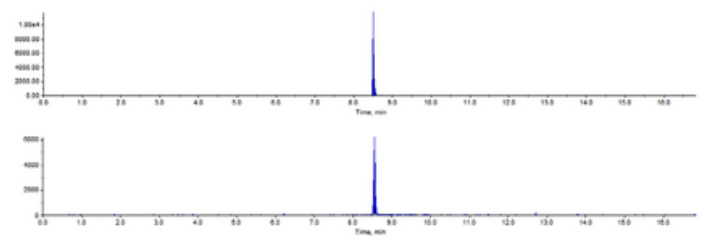

C

D

\section{Figure 2}

Representative MRM chromatograms of luteoloside (1), apigetrin (2), hesperidin (3) and quercitrin (4, IS) in rat plasma (A. blank plasma; B. blank plasma added with the three analytes at LLOQ and IS; C. plasma sample at $1 \mathrm{~h}$ after oral administration of STAE; D. plasma sample at $1 \mathrm{~h}$ after oral administration of STAE and STVO)
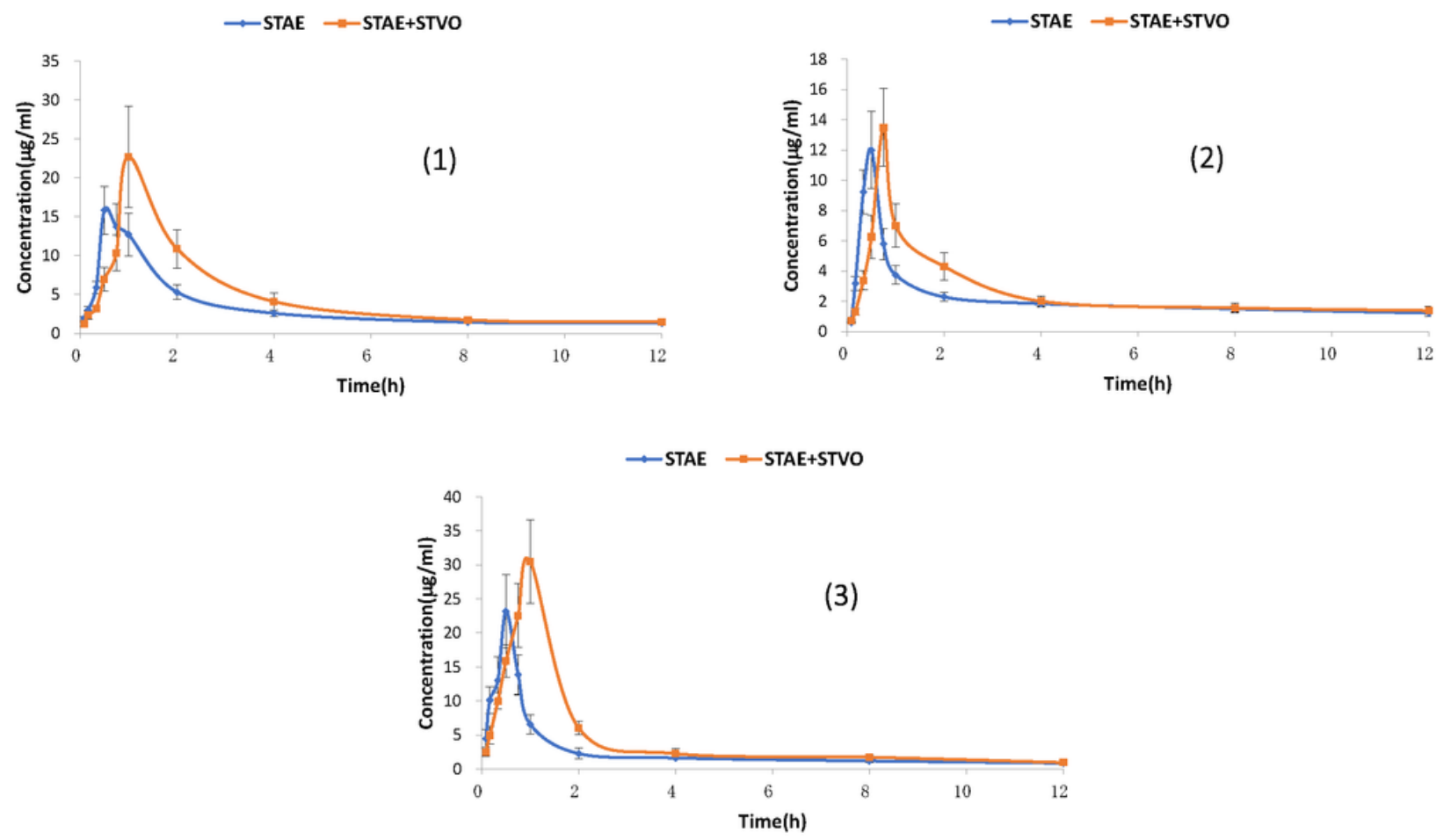


\section{Figure 3}

Time-dependent curves of luteoloside (1), apigetrin (2) and hesperidin (3) in STAE group and STAE+STVO group
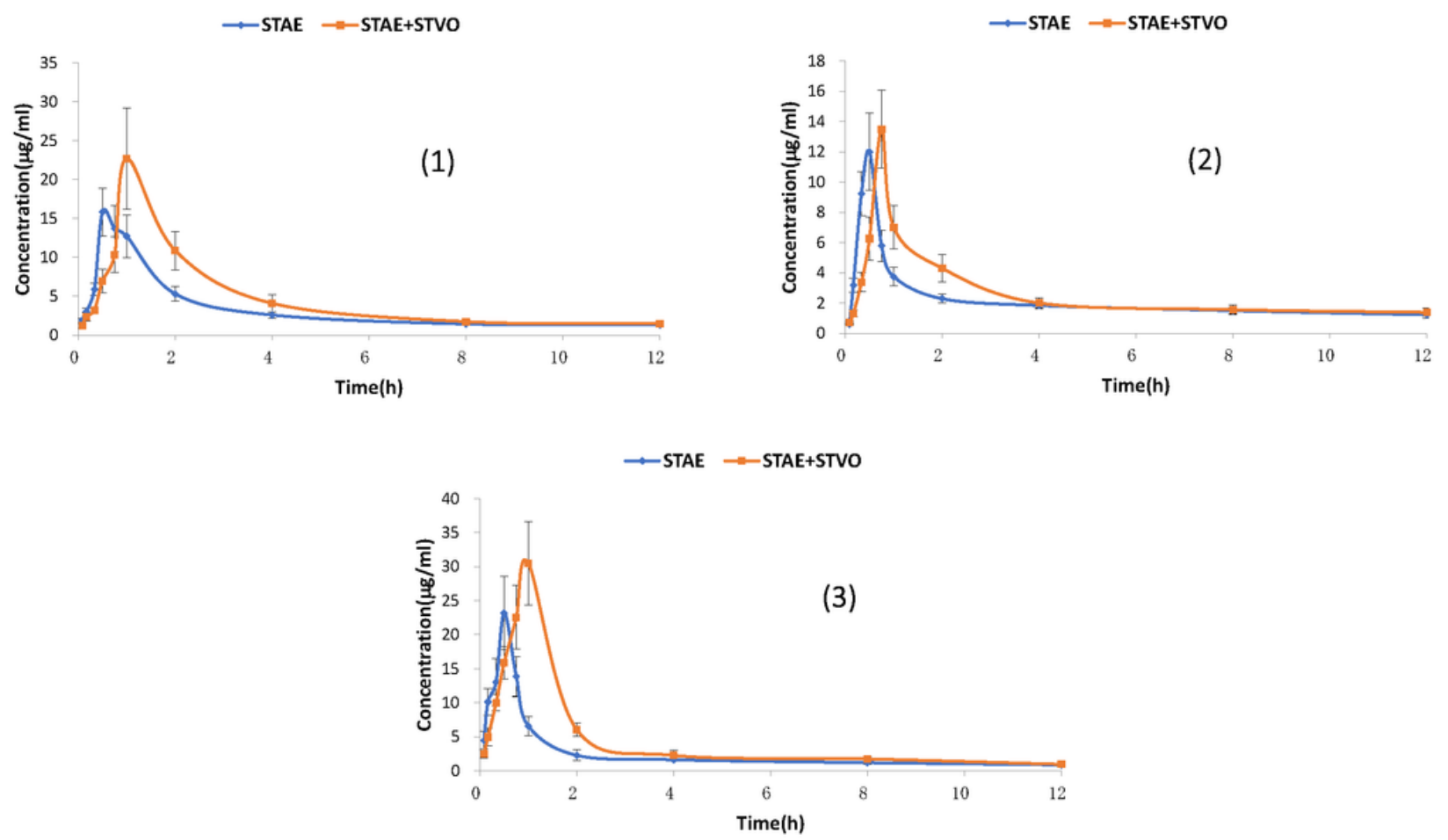

\section{Figure 3}

Time-dependent curves of luteoloside (1), apigetrin (2) and hesperidin (3) in STAE group and STAE+STVO group 